\title{
Editorial
}

\section{Skeletal Muscle Should Not Be Overlooked}

\section{Ji A Seo}

Division of Endocrinology and Metabolism, Department of Internal Medicine, Korea University Ansan Hospital, Korea University College of Medicine, Ansan, Korea

Overweight and obesity are defined as abnormal or excessive fat accumulation that presents a risk to health, including development of cardiometabolic abnormalities. Because direct measurement of body composition is difficult in routine clinical settings, body mass index (BMI) instead of fat mass is used often to diagnose obesity. BMI is a reasonably good measure of general adiposity, and many epidemiologic studies have reported that obesity, defined by BMI, is a significant risk factor for increased risk of cardiovascular disease (CVD) and mortality $[1,2]$. However, BMI has a major limitation in that it dose not discriminate between fat mass and lean body mass. Evidence suggests that skeletal muscle, which accounts for most of lean body mass, is beneficial for cardiometabolic health [3]. Metabolism and activity, which directly influence CVD risk factors, can be driven by skeletal muscle.

Over the past decades, two phenomena have shown that the relationship between obesity and increased health risk is not simple. First, observations that a subgroup of individuals with obesity showed significantly lower obesity-related cardiometabolic abnormalities led to the concept of 'metabolically healthy obesity (MHO).' MHO is characterized by lower liver and visceral fat mass, higher leg fat content, greater cardiorespiratory fitness and physical activity, better insulin sensitivity, lower levels of inflammatory markers, and normal adipose tissue function compared to metabolically unhealthy obesity [4]. Although there is no universally accepted definition of MHO, normal glucose and lipid parameters, absence of hypertension, and BMI-defined obesity have been proposed as criteria. Accumulating evidence in recent decades supports that MHO involves increased cardiometabolic risk and is not a benign con- dition [4]. In Korean adults, even in people with persistent MHO, CVD incidence was more than twice that of people with metabolically healthy normal weight [5]. Therefore, personalized and risk-stratified obesity treatment for $\mathrm{MHO}$ subjects is required.

The second phenomenon is the J or U-shaped relation between BMI and mortality, the so-called 'obesity paradox.' The exact mechanism responsible for this association is unknown, and many researchers criticize possible reverse causation due to illness-induced weight loss and the effects of confounding factors. A recent report showed low lean body mass, rather than fat mass, to be the main factor driving the 'obesity paradox' for mortality in the lower BMI range [6]. The results suggest that low lean body mass is a more important contributor to health risk in nonobese people than in obese people.

In this issue of Diabetes \& Metabolism Journal, Cho et al. [7] investigated the association of sarcopenia (defined by low muscle mass) with CVD risk according to obesity phenotype in the National Health and Nutrition Examination Survey population. As expected, sarcopenia was correlated with increased CVD risk in each obesity phenotype. Interestingly, subjects with metabolically healthy normal weight with sarcopenia showed similar increased association with CVD compared to subjects with $\mathrm{MHO}$ without sarcopenia. These results suggest skeletal muscle mass as an important confounding factor to evaluate the relationship between obesity phenotype and cardiometabolic complications, and the limitation of BMI-defined obesity should be considered. In addition, the difference in CVD risk between sarcopenia and no sarcopenia groups seems clearer in subjects without metabolic risk factors. Heter-
Corresponding author: Ji A Seo (iD https://orcid.org/0000-0002-1927-2618 Division of Endocrinology and Metabolism, Department of Internal Medicine, Korea University Ansan Hospital, Korea University College of Medicine, 123 Jeokgeum-ro, Danwon-gu, Ansan 15355, Korea

E-mail: seojia@korea.ac.kr
This is an Open Access article distributed under the terms of the Creative Commons Attribution Non-Commercial License (https://creativecommons.org/licenses/by-nc/4.0/) which permits unrestricted non-commercial use, distribution, and reproduction in any medium, provided the original work is properly cited. 
ogenous characteristics of subjects in the same obesity phenotype comprise more than just skeletal muscle. The prevalence or severity of comorbid diseases such as diabetes and hypertension might have a greater impact on CVD risk than dose obesity phenotype [8]. Because the analyses were performed in a cross-sectional design, they could not evaluate a causal effect. Indeed, the association between low muscle mass and increased CVD risk was possibly due to reverse causation from the impact of CVD on muscle loss. Prospective studies are necessary to clarify the bidirectional association between sarcopenia and CVD risk according to obesity phenotype.

Nevertheless, Cho et al.s [7] result reminds us that considering muscle is important for CVD risk evaluation in medical care or in epidemiologic studies regardless of the presence of obesity or metabolic abnormalities. In this context, they demonstrated possible usefulness of "A Body Shape Index" (ABSI) as a simple indicator of muscle and fat mass in general clinical settings with limited equipment. ABSI seems to supplement BMI to estimate CVD risk especially in people with normal BMI. The effect of ABSI on CVD risk stratification by obesity phenotype should be demonstrated in prospective studies.

Meanwhile, lower muscle strength and poor muscle quality rather than decreased muscle mass are drawing more attention as causes of increased health risks [9]. Most recent consensus reports have included low muscle strength or low muscle function as an essential component for definition and diagnosis of sarcopenia [10,11]. Moreover, muscle strength decreases after a peak in young adulthood gradually but faster than that in muscle mass [12]. Not only measurement of muscle quantity, but also active efforts to evaluate muscle strength (for example, measurement of grip strength or chair stand test) might be necessary to properly access the association between sarcopenia and CVD risk in the context of body phenotype.

Body phenotype is changing continuously. In a Korean population-based cohort, $36 \%$ of metabolically healthy normal weight subjects and $51 \%$ of MHO subjects changed to metabolically unhealthy phenotype after 10 years of follow-up [5]. Regardless of the current obesity phenotype, it is important to reduce body fat and maintain healthy skeletal muscles.

\section{CONFLICTS OF INTEREST}

No potential conflict of interest relevant to this article was reported.

\section{REFERENCES}

1. Berrington de Gonzalez A, Hartge P, Cerhan JR, Flint AJ, Hannan L, MacInnis RJ, et al. Body-mass index and mortality among 1.46 million white adults. N Engl J Med 2010;363:2211-9.

2. Khan SS, Ning H, Wilkins JT, Allen N, Carnethon M, Berry JD, et al. Association of body mass index with lifetime risk of cardiovascular disease and compression of morbidity. JAMA Cardiol 2018;3:280-7.

3. Tyrovolas S, Panagiotakos D, Georgousopoulou E, Chrysohoou C, Tousoulis D, Haro JM, et al. Skeletal muscle mass in relation to 10 year cardiovascular disease incidence among middle aged and older adults: the ATTICA study. J Epidemiol Community Health 2020;74:26-31.

4. Blüher M. Metabolically healthy obesity. Endocr Rev 2020;41: 405-20.

5. Kim NH, Seo JA, Cho H, Seo JH, Yu JH, Yoo HJ, et al. Risk of the development of diabetes and cardiovascular disease in metabolically healthy obese people: the Korean Genome and Epidemiology Study. Medicine (Baltimore) 2016;95:e3384.

6. Lee DH, Keum N, Hu FB, Orav EJ, Rimm EB, Willett WC, et al. Predicted lean body mass, fat mass, and all cause and cause specific mortality in men: prospective US cohort study. BMJ 2018;362:k2575.

7. Cho HW, Chung W, Moon S, Ryu OH, Kim MK, Kang JG. Effect of sarcopenia and body shape on cardiovascular disease according to obesity phenotypes. Diabetes Metab J 2021;45:20918.

8. Sung KC, Ryu S, Cheong ES, Kim BS, Kim BJ, Kim YB, et al. All-cause and cardiovascular mortality among koreans: effects of obesity and metabolic health. Am J Prev Med 2015;49:62-71.

9. Cruz-Jentoft AJ, Sayer AA. Sarcopenia. Lancet 2019;393:263646.

10. Cruz-Jentoft AJ, Bahat G, Bauer J, Boirie Y, Bruyere O, Cederholm T, et al. Sarcopenia: revised European consensus on definition and diagnosis. Age Ageing. 2019;48:16-31.

11. Chen LK, Liu LK, Woo J, Assantachai P, Auyeung TW, Bahyah KS, et al. Sarcopenia in Asia: consensus report of the Asian Working Group for Sarcopenia. J Am Med Dir Assoc 2014;15: 95-101.

12. Dodds RM, Syddall HE, Cooper R, Benzeval M, Deary IJ, Dennison EM, et al. Grip strength across the life course: normative data from twelve British studies. PLoS One 2014;9:e113637. 